\title{
Location Manager based Handover Method for LEO Satellite Networks
}

\author{
Soumya Das \\ Department of E.T.C.E \\ Bengal Institute of Tech. \\ W.B.U.T \\ Suman Kumar Sikdar \\ M. Tech in C.S.E \\ Jadavpur University
}

\author{
Debabrata Sarddar \\ (Asst. Proff.) \\ Department of C.S.E. \\ Kalyani University \\ Sougata Chakraborty \\ M.Tech in C.S.E. \\ Jadavpur University
}

\author{
Dipsikha Ganguly \\ Department of E.T.C.E. \\ B.P. Poddar I.M.T. W.B.U.T \\ Kunal Hui \\ M. Tech in C.S.E. \\ Jadavpur University
}

\begin{abstract}
Low Earth Orbit (LEO) satellite made a great impact to the scientists towards the end of the previous decade because of its some interesting features such as low propagation delay, low power requirements and the ability to communicate with handheld terminals. That's why future satellite networks are now conceived as complementary rather than competitive to terrestrial networks. But as the speed of LEO satellite is higher than Mobile Nodes (MN) and earth's speed, the no of handover occurrence is more which degrades the overall communication quality. Also the call blocking probability and forced call termination probability is more. To solve these problems, a number of handover methods have been proposed by different scientists. Here we have proposed a fast method for handover named Location Manager Based Handover method for LEO satellite networks where we have use Location Manager (LM) for reducing the scanning time.

LM is used to store all the mobility pattern of all the satellites. To know how this method works, we run a set of simulations for handover latency, handover throughput and call blocking probability for our proposed method with the standard methods. Results show that this algorithm can significantly improve the overall communication performance.
\end{abstract}

\section{Keywords}

LEO satellite, handover latency, location manager

\section{INTRODUCTION}

The demand for global broadband telecommunication services is growing rapidly in next generation mobile satellite systems, and they are expected to provide anytime-anywhere communication services [1][2]. Global coverage is the most important advantage of LEO satellite networks. Low earth orbit satellites rotate around the Earth at altitudes ranging from 500 to $2000 \mathrm{~km}$. They have important properties like low propagation delay and low equivalent isotropic radiated power (EIRP) requirements for hand-held devices. So LEO satellites make it possible to support real-time interactive multimedia traffic

for their users with better quality-of-service (QoS) [3] guarantees.

Quality-of-service parameters include bandwidth, delay, jitter, call blocking probability, call dropping probability etc.

In cellular telecommunications, the term handover or handoff refers to the process of transferring an ongoing call or data session from one channel connected to the core network to another. In satellite communications it is the process of transferring satellite control responsibility from one earth station to another without loss or interruption of service.

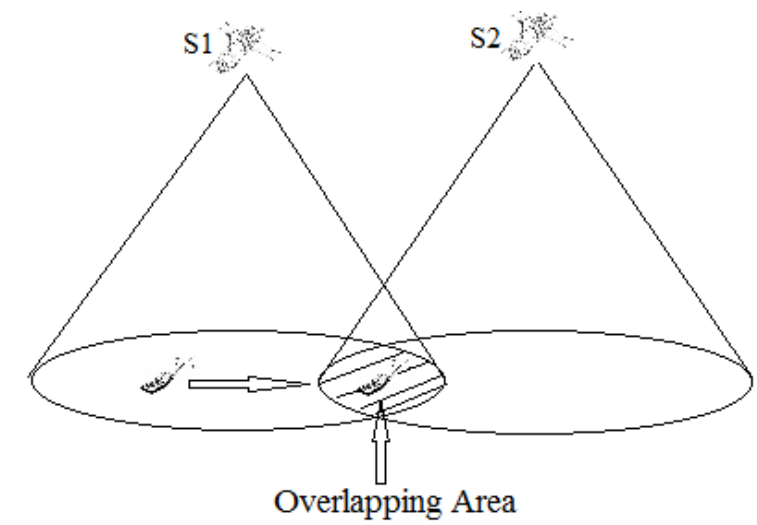

Figure: 1 Handover Occurrence

A Hard Handover is one in which the channel in the source cell is released and only then the channel in the target cell is engaged. Thus the connection to the source is broken before or 'as' the connection to the target is made - for this reason such handovers are also known as break-before-make. Hard handovers are intended to be instantaneous in order to minimize the disruption to the call. When the mobile is between base stations, then the mobile can switch with any of the base stations, so the base stations bounce the link with the mobile back and forth. [4]

A Soft Handover is one in which the channel in the source cell is retained and used for a while in parallel with the channel in the target cell. In this case the connection to the target is established before the connection to the source is broken, hence this handover is called make-before-break. The interval, during which the two connections are used in parallel, may be brief or substantial. Soft handovers may involve using connections to more than two cells: connections to three, four or more cells can be maintained by one phone at the same time. The latter is more advantageous, and when such combining is performed both in the downlink (forward link) and the uplink (reverse link) the handover is termed as softer. Softer handovers are possible when the cells involved in the handovers have a single cell site [5].

Scanning: When a mobile station is moving away from its current satellite, it initiates the handoff process when the received signal strength and signal-to-noise-ratio have decreased below the threshold level. The MN now begins the scanning to find new satellite. It can either opt for a passive 
scan (where it listens for beacon frames periodically sent out by satellites) or chose a faster active scanning mechanism wherein it regularly sends out probe request frames and waits for responses for $\mathrm{T}_{\mathrm{MIN}}$ (min Channel Time) and continues scanning until $\mathrm{T}_{\text {MAX }}$ (max Channel Time) if at least one response has been heard within $\mathrm{T}_{\mathrm{MIN}}$. Thus, $\mathrm{n} * \mathrm{~T}_{\mathrm{MIN}} \leq$ time to scan $n$ channels $\leq n * T_{M A X}$. The information gathered is then processed so that the MN can decide which Satellite to join next. The total time required until this point constitutes $90 \%$ of the handoff delay [6][7].

Authentication: Authentication is necessary to associate the link with the new satellite. Authentication must either immediately proceed to association or must immediately follow a channel scan cycle. In pre-authentication schemes, the $\mathrm{MN}$ authenticates with the new satellite immediately after the scan cycle finishes.[8][9]

Re-Association: Re-association is a process for transferring associations from old satellite to new one. Once the $\mathrm{MN}$ has been authenticated with the new satellite, re-association can be started. Previous works has shown re-association delay to be around 1-2 ms. The range of scanning delay is given by:-

$\mathrm{N} \times$ Tmin _ Tscan _ $\mathrm{N} \times \mathrm{Tmax}$

Where $\mathrm{N}$ is the total number of channels according to the spectrum released by a country, Tmin is Min Channel Time, Tscan is the total measured scanning delay, and Tmax is Max Channel Time. Here we focus on reducing the scanning delay by minimizing the total number of scans performed.[10],[11]. In this paper we have proposed a new handover technique which reduces the handover latency. Here we used the LM which is a database manager and supports the management of whole system.

The paper is organised as follow: in the second section we have described the related works on handover management. In the third section we have described the details of LMBHO. In the forth section the simulation results of both our method and standard methods. In the next section we conclude the whole paper and finally a future work is mention regarding this paper in section six.

\section{RELATED WORK}

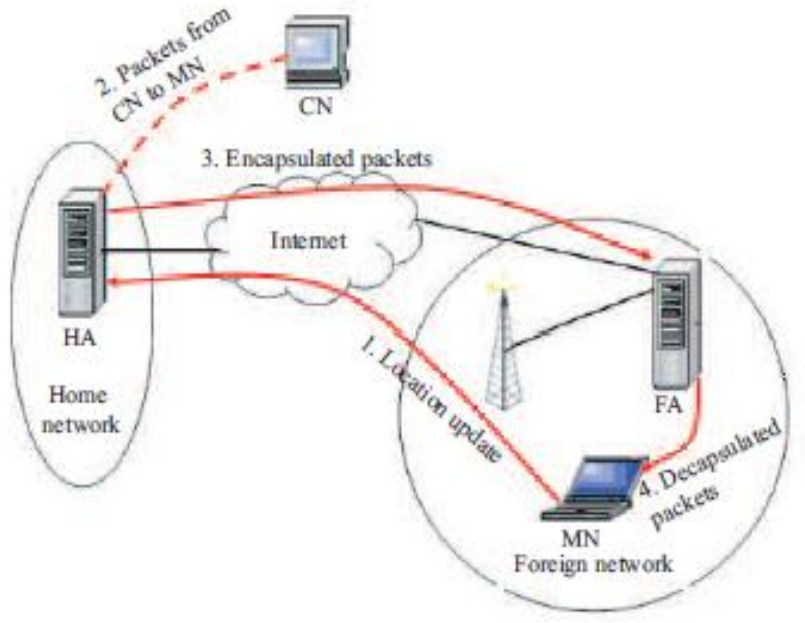

Figure 2: Handover Flow of Mobile IP

The most widely used protocol for handover in satellite is MIP [12]. It is proposed by The Internet engineering task force (IETF) to handle mobility of internet hosts for mobile data communications. MIP is based over the concept of Home
Agent (HA) and Foreign Agent (FA) for delivering of packets from one $\mathrm{MN}$ to $\mathrm{CN}$. It is basically completed by four steps.

i) When handover begins $\mathrm{MN}$ registers itself in FA and waits for allocation of channels in FA and updates its location in HA directory.

ii) The packets are sent to HA and HA encapsulate it.

iii) Encapsulated packets are sent to The FA.

iv) FA decapsulate those packets and sent it to MN.

The main drawback of this protocol is

$>$ High handover latency

$>$ High packet lost rate

$>$ Insufficient routing path

$>$ Conflicts with network security solution

Another method is Seamless handover management scheme (SeaHO-LEO) [13], [14] proposed by Aysegul et al in 2006.

It reduces packet loss and handover latency. It is describes as follows

A. Calculate a new IP

B. Send handover preparation request to current satellite

C. Start to use new IP to send data packets

D. CN starts to use new satellite

SeaHO-LEO provides efficient utilization of network bandwidth because of the absence of tunnelling and also does not need any change in existing internet infrastructure.

The main disadvantage of this process is high messaging traffic.

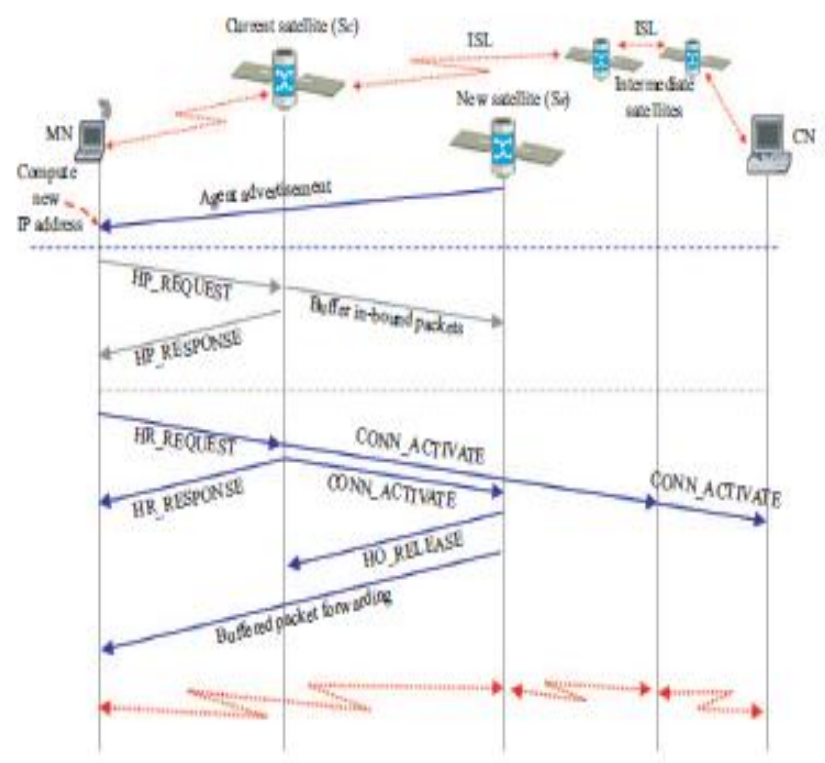

Figure 3: Signalling Flow of SeaHO-LEO

Another method to remove high messaging traffic is Pattern based handover management (PatHO-LEO) [13],[14]. It describes as follows 


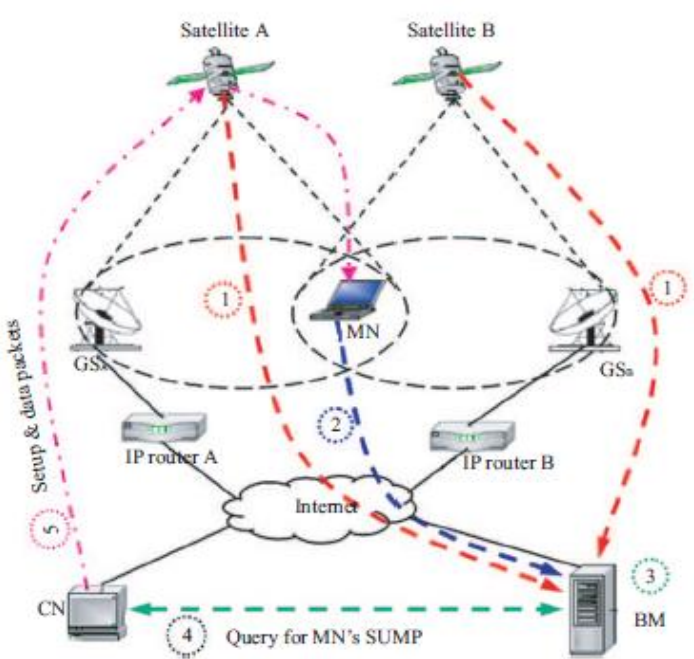

Figure 4: Handover scenario in PatHO-LEO

$\checkmark \quad$ Satellite register to BM.

$\checkmark$ MN registers to BM.

$\checkmark$ BM establishes the satellite and user mobility pattern (SMUP) table.

$\checkmark \quad \mathrm{CN}$ and BM establish connection.

$\checkmark \mathrm{CN}$ sends data packets to MN.

But the main drawback of PatHO-LEO is that

i) Every user should have a specific mobility pattern in a specific period of time. A user can have more than one mobility pattern. But when it violets its mobility pattern the handover process will be either in SeaHO-LEO or MIP.

ii) The no of user who do not have a specific mobility pattern in a week is increasing day by day like salesman, LIC worker who have to go different place at different time in a week.

Also there are other mobility management protocols such as Transport layer seamless handoff schemes for space networks (TraSH-SN) [15], paging in mobile IP (P-MIP) [16], and cellular IP [17]. These methods will not be covered in this paper.

\section{PROPOSED WORK}

In our proposed method we assume that the communication is done in between two $\mathrm{MN}$ or in between a $\mathrm{MN}$ and a $\mathrm{CN}$. So our method supports voice call, video call, messaging as well as file downloading, file uploading and web browsing.

The standard method for handover in LEO satellite is MIP. But the main disadvantage of MIP handover is that it has high handover latency and high packet data lost rate. This drawback is omitted in the SeaHO-LEO proposed by Aysegul et al. But SeaHO-LEO has some drawback such as high handover messaging traffic. This disadvantage is also omitted by PatHO-LEO method. But the main drawback of PatHOLEO is that described above

To eliminate this drawback we propose a new method for satellite handover named Location Manager Based Handover (LMBHO) method where we have introduced the concept of Location Manager (LM).

As we know that the movement of all satellites are deterministic. That means every day the satellites cover same region at the same time. So if we save this data in our database we can find the position of any satellite in time in a day. This data is stored in the location Manager. Thus at the time of handover, the MN do not scan for the new satellites as the location of the new satellite can be found from location manager.

The following three terminologies which we will use throughout this paper

$>$ Current Satellite: The satellite through which the present connection is going i.e. the satellite through which $\mathrm{MN}$ is connected before handover.

$>$ New Satellite: The satellite through which the MN will be connected after handover.

$>$ Adjacent Satellite: If the connection is going through more than one satellite then the concept of adjacent satellite arises. It is the satellite which forwards the data to the current satellite before handover and new satellite after handover.

In our proposed method we assume that the $\mathrm{MN}$ is equipped with GPS so that the positions of the MN can be found at the time of handover.

Now we will define 3 levels for handover based on the received signal strength and signal-to-noise ratio $(\mathrm{SS} \& \mathrm{~S} / \mathrm{N})$.

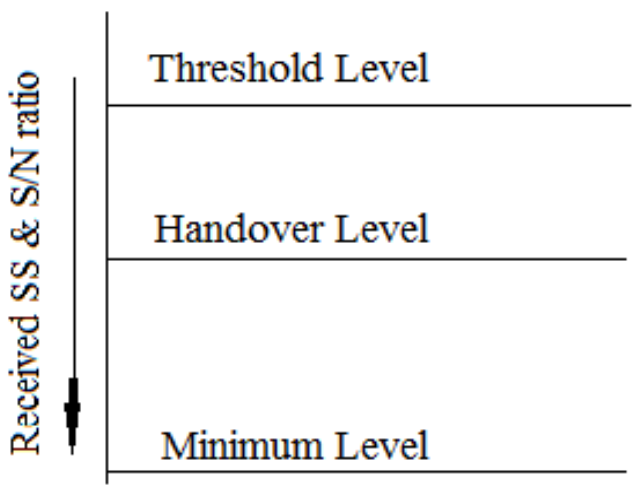

Fig 5 levels for handover

A. Threshold Level: Whenever the received $(\mathrm{S} / \mathrm{N})$ crosses this level the LMBHO method begins.

B. Handover level: whenever the received SS \& S/N crosses this level, the handover preparation step is completed and the handover completion step begins.

C. Minimum level: Whenever the received SS and S/N crosses this level the connection is lost. So this is the minimum level for the connection. The handover must be completed before $\mathrm{MN}$ crosses this level.

The LMBHO has the following steps

A. Handover Preparation: In this step the necessary preparation for handover is done. This step is completed before MN crosses the Handover level.

B. Handover Completion: In this step the handover is completed using the new satellite. This step must be completed before received $\mathrm{SS}$ and $\mathrm{S} / \mathrm{N}$ crosses the minimum level.

Initial Set Up: Initially all the positions of the satellite with corresponding time are saved in location manager. All the satellite can use this database form LM whenever the handover begins.

Step 1: The handover generally begins when the $\mathrm{MN}$ enters the overlapping coverage area of the two adjacent satellites. But it is not possible for $\mathrm{MN}$ to find whether it has moved to 
this overlapped area. So MN continuously checks the received $\mathrm{SS} \& \mathrm{~S} / \mathrm{N}$ crosses the threshold level of handover. If it crossed then the handover begins.

Step 2: Now as soon as MN finds that the received SS \& S/N crosses the threshold level, it sends an EQ_MSG to LM enquiring about the name of satellites covers that region at that time. EQ_MSG contains the position of $\mathrm{MN}$ at that time and the IP address of the current satellite.

Step 3: After receiving the EQ_MSG, LM finds the list of satellites available at that time form its database other than current satellite. Then LM responses with a RS_MSG which contains the list IP addresses of the satellites available at that time.

Step 4: After receiving the RS_MSG, MN sends a QS_MSG to all the satellites in the RS_MSG to know the signal strength and the channel capacity of that satellites at that time.

Step 5: As soon as any satellite receives QS_MSG it replies back to that MN with RP_MSG which contains the signal strength and channel capacity at that time.

In the meantime MN continuously checks if the received SS $\& \mathrm{~S} / \mathrm{N}$ crosses the handover level. If it crosses then it starts the handover completion steps which are described as follows

Step 6: Now MN selects the best satellite available on the basis of Quality of Service (QOS) parameters which are

- Maximum service time

- Maximum number of free channels

- Minimum distance

It also includes the signal strength and the channel capacity of the satellites. A specific algorithm has to be developed for selection (In our future work).

Step 7: Now MN sends a handover request HO_REQ to the new satellite which contains the IP address of the MN, IP address of the current satellite and IP address of the adjacent satellite.

Step 8: As soon as the new satellite receives the HO_REQ it sends a connection release message CONN_REL to the current satellite to release the connection from $\mathrm{MN}$. It simultaneously sends a connection activate message CONN_ACT to the adjacent satellite which contains the IP address of the current satellite.

Step 9: whenever adjacent satellite receives the CONN_ACT message it sends the next data to the new satellite and the new satellite forwards it to the MN. Meanwhile the current satellite releases the connection as soon as it receives the CONN_REL message. Now the connection is made by the new path and the handover is completed.

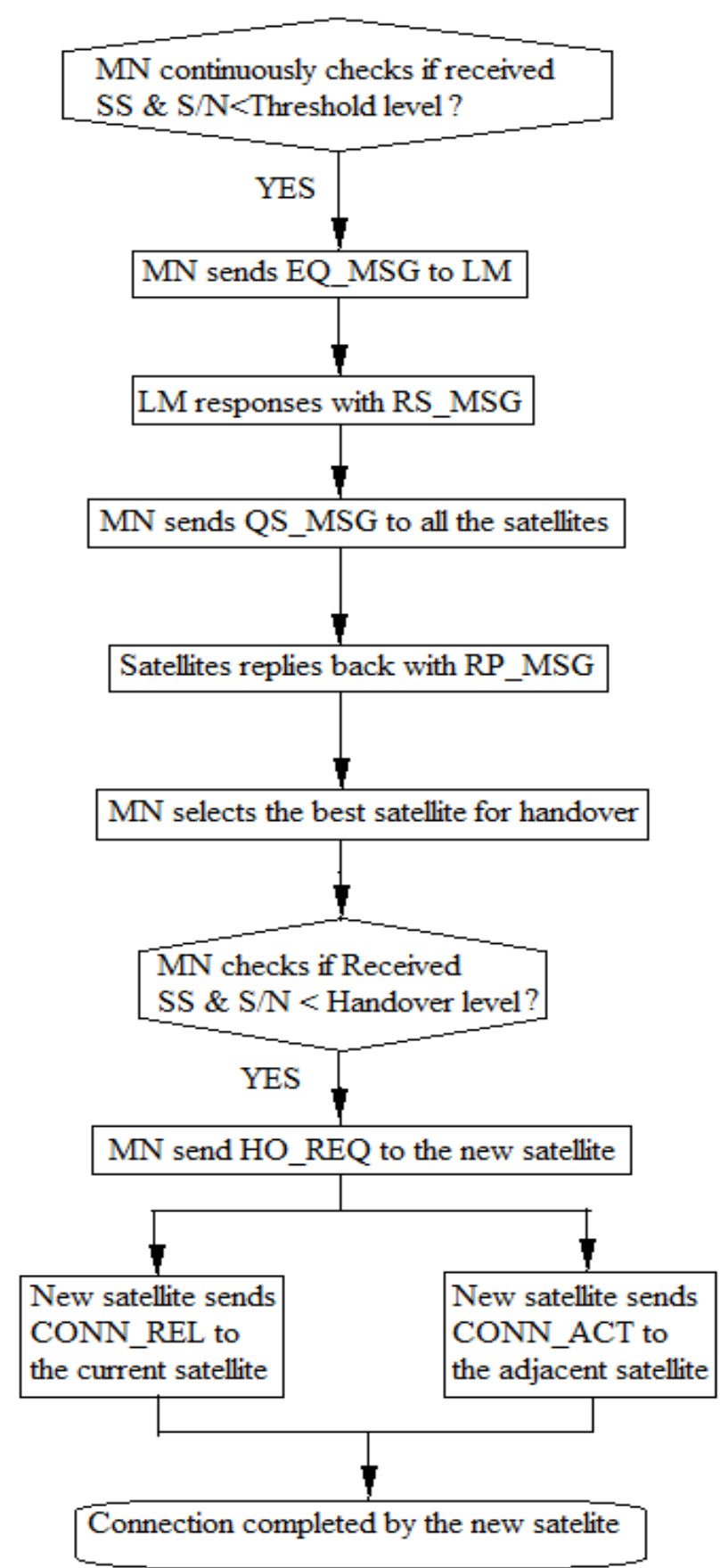

Fig: 6 flow chart of LMBHO method

The advantages of this proposed method are

A. This method reduces the scanning time for searching a new satellite. Actually the scanning time equal to zero as no scanning is required for finding the new satellite. The list of available satellites can be found by sending only an EQ_MSG to LM.

B. The high messaging traffic has been reduced in this method

C. As this method uses only the patterns of satellites which is not subjected to change so this method can be used everywhere unlike PatHO-LEO which cannot be used where user does not have any specific mobility pattern. 
D. This method can be used for static $\mathrm{CN}$ as well as movable another MN. The signal flow can be one sided or both sided.

\section{SIMULATION RESULTS}

Now we will evaluate the performance of our proposed work. We compare our proposed work to MIP and SeaHO_LEO.

Each algorithm is evaluated by analysing MN's average handover throughput, call blocking probability and handover latency. The simulation results were run on MATLAB 7.8 in a designed virtual environment.

The simulation environment is created by setting the following parameters.

\begin{tabular}{|l|l|}
\hline $\begin{array}{l}\text { Satellite coverage area } \\
\text { radius }\end{array}$ & $700[\mathrm{~km}]$ \\
\hline Satellite ground Speed & $7[\mathrm{~km} / \mathrm{sec}]$ \\
\hline $\begin{array}{l}\text { Mobile Node speed } \\
\text { Number of nodes reside } \\
\text { in the coverage area }\end{array}$ & $17[\mathrm{~km} / \mathrm{sec}](60 \mathrm{~km} / \mathrm{hr})$ \\
\hline \begin{tabular}{l} 
Time in footprint \\
\hline
\end{tabular} & $5 \mathrm{~min}$ \\
\hline
\end{tabular}

Here we assume that the generated traffic is according to the Poisson distribution function.

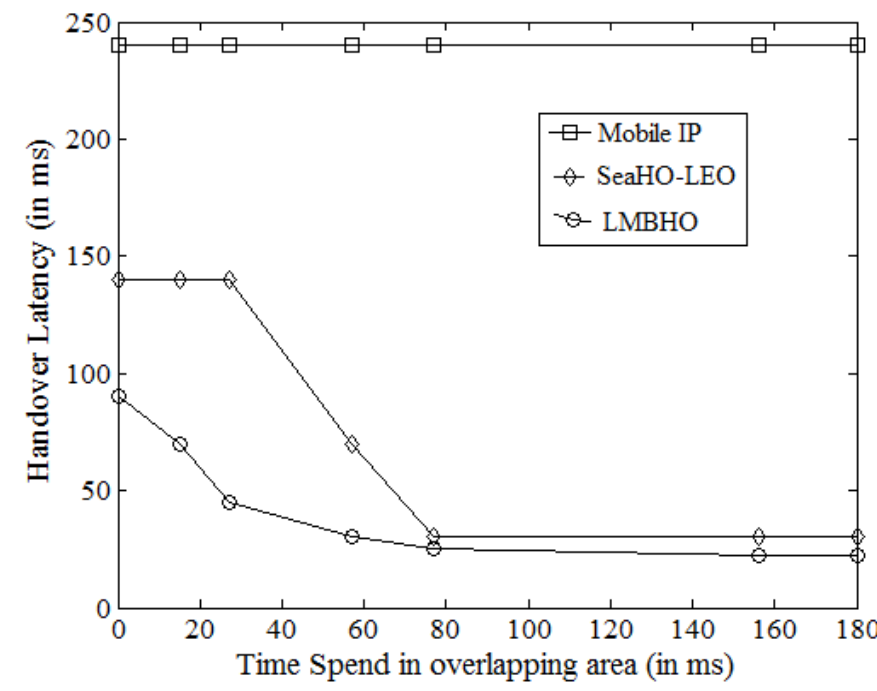

Fig 7 Handover Latency

In figure 7 we have evaluated the average handover latency of different algorithms. Handover latency is the time taken for establishing a new connection current satellite to new satellite. In other words handover latency is the time interval between the last data packet received from the current satellite and the first data packet received from the new satellite.

In the figure we see that the handover latency of MIP is very high, around 240ms. This is because in MIP during handover, $\mathrm{MN}$ has to send location update message to it's HA to associate its home address and CoA. This binding update process is very time consuming. Besides, MIP is unable of receiving packets in flight during this handover process. In SeaHO_LEO, whenever old satellite receives a
HR_REQUEST message, it establishes a virtual communication path between the new satellite and $\mathrm{MN}$ by the help of ISL between it and new satellite (approximately $20 \mathrm{~ms}$ ). In LMBHO the no of new satellite is already saved into the LM's database. So the current satellite does not search for a new satellite. Just sends a QS_MSG to all that satellite. So the scanning time is reduced to zero. Thus the handover latency is also reduced (approximately 14ms).

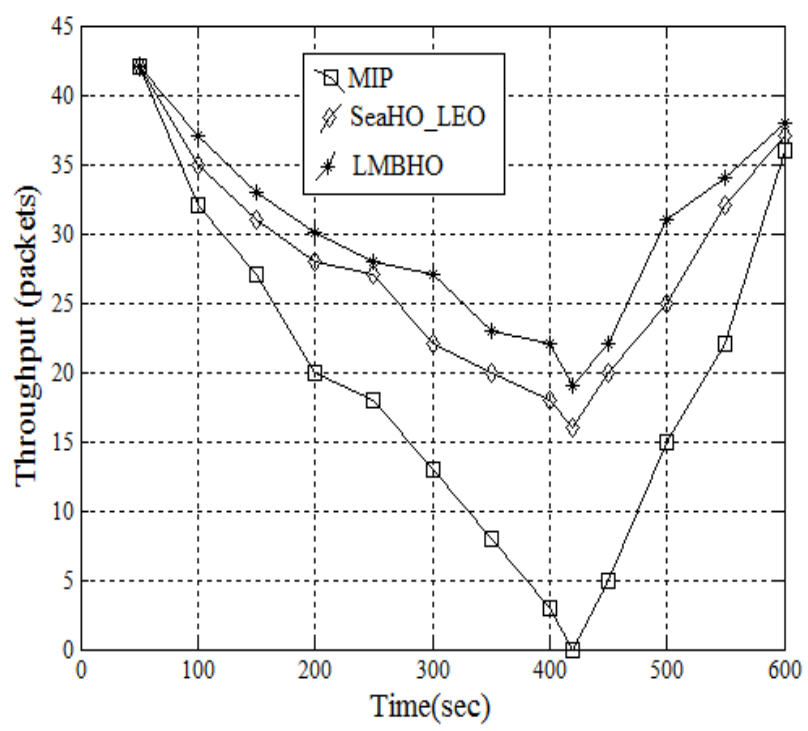

Fig 8 MN's handover throughput

Fig 8 shows the MN's average handover throughput during any handover process for MIP, SeaHO_LEO and our proposed work.

In MIP, due to the tunnelling between HA and FA, throughput of the channel between $\mathrm{MN}$ and $\mathrm{CN}$ converges to zero during handover. When the handover model is completed, the throughput reaches a reasonable value. The throughput of SeaHO-LEO is better than MIP during handover as it does not reach to zero.

In LMBHO the handover throughput is better than SeaHO_LEO as whenever the adjacent satellite receives CONN_ACT message, it forwards next packed data to the new satellite.

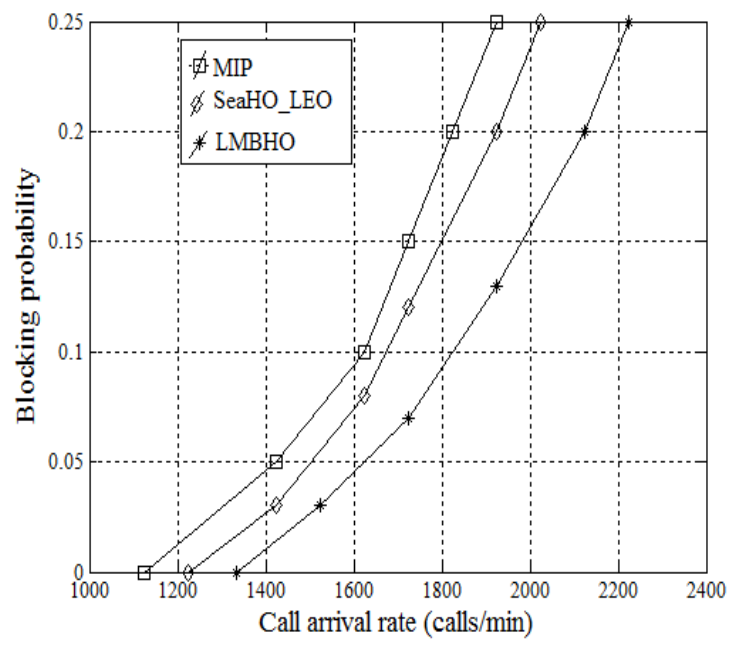

Fig 9 Blocking probability of a handover call 
In figure 9 we have evaluated the blocking probability of a handover call. The blocking probability of MIP is highest. SeaHO_LEO has almost same amount of blocking probability. The blocking probability of LMBHO is lowest.

\section{CONCLUSION}

In this paper we have proposed a new method for handover named LMBHO based on the concept of Location Manager.

We first described the future aspects of LEO satellites and its advantages. Then we defined handover and different types of handover. After that we explained the details of scanning. Then we explained in brief some of the standard protocols MIP, SeaHO_LEO, PatHO_LEO and also mentioned their drawbacks. The details of our proposed method LMBHO is given in the next section and we also mentioned its advantages. An extensive set of simulations were performed in a virtual environment created in Matlab. The simulation results based on the handover latency, handover throughput and call blocking probability shows that our method can significantly increase the quality of communication by decreasing the handover latency and call blocking probability and increasing the average handover throughput.

\section{FUTURE WORK}

In future we will try to find out an algorithm for selecting the best satellite. Also we will study how to decrease the forced call termination probability.

\section{REFERENCES}

[1] Enrico D R, Fantacci R, Giambene G. Characterization of user mobility in low earth orbiting mobile satellite systems. Wireless Networks, 2000, 6(3): $165-179$

[2] Akyldiz I F, Jeong S H. Satellite ATM networks: A survey. IEEE Communications, 1997, 35(7): 30-43

[3] E. Papapetrou and F.-N. Pavlidou, -QoS handover management in LEO/MEO satellite systems, $\|$ Wireless Personal Communications, vol. 24, no. 2, pp. 189-204, February 2003.

[4] A. Jamalipour, -Satellites in IP networks, $\|$ in Wiley Encyclopaedia of Telecommunications, vol. 4, Wiley, 2002, pp. 2111-2122

[5] S. L. Kota, P. A. Leppanen, and K. Pahlavan, Broadband Satellite Communications For Internet Access, Kluwer Academic Publishers, 2004

[6] Debabrata Sarddar, Shubhajeet Chatterjee, Ramesh Jana, Shaik Sahil Babu, Hari Narayan Khan, Utpal Biswas and M.K.Naskar, - Fast Handoff Implementation by using Curve Fitting Equation With Help of GPS\|, International Journal of Computer Science issues (IJCSI) Vol. 08, Issue 03, pp. 535-542, No. 1, May 2011, ISSN (Online): 1694-0814.

[7] Debabrata Sarddar, Shubhajeet Chatterjee, Ramesh Jana, Hari Narayan Khan, Shaik Sahil Babu, Hari Narayan Khan, Utpal Biswas and M.K.Naskar, - Minimization of Handoff Latency by Vector Analysis Methodll, International Journal of Computer Science issues (IJCSI) Vol. 08, Issue 03, pp. 570-576, No. 1, May 2011, ISSN (Online): 1694-0814.

[8] Debabrata Sarddar, Soumya Das, Dipsikha Ganguli, Kalyan Kumar Das, Sougata Chakraborty, Kunal Hui and Mrinal Kanti Naskar. Article: A New Method for Controlling Mobility Management Cost of PatHO-LEO satellite and Mobile IP Network. International Journal of Computer Applications 37(7):32-38, January 2012.
Published by Foundation of Computer Science, New York, USA.

[9] Debabrata Sarddar, Shubhajeet Chatterjee, Ramesh Jana, Hari Narayan Khan, Shaik Sahil Babu, Hari Narayan Khan, Utpal Biswas and M.K.Naskar, - Minimization of Handoff Latency by Vector Analysis Methodll, International Journal of Computer Science issues (IJCSI) Vol. 08, Issue 03, pp. 570-576, No. 1, May 2011, ISSN (Online): 1694-0814.

[10] Debabrata Sarddar, Shubhajeet Chatterjee, Ramesh Jana, Shaik Sahil Babu, Hari Narayan Khan, Utpal Biswas and M.K.Naskar, - Minimization of Handoff Latency by Distance Measurement Methodll, International Journal of Computer Science issues (IJCSI) Vol. 08, Issue 02, pp. 283-289, March 2011, ISSN(Online): 1694-0814.

[11] Debabrata Sarddar, Soumya Das, Dipsikha Ganguli, Sougata Chakraborty, Kunal Hui, Kalyan Kumar Das and Mrinal Kanti Naskar. Article: A New Method for Fast and Low Cost Handover in Leo Satellites. International Journal of Computer Applications 37(7):39-45, January 2012. Published by Foundation of Computer Science, New York, USA

[12] C. Perkins, -IP mobility support,\| RFC 2002, IEFT, Oct. 1996, available at http://www.ietf.org/rfc/rfc2002.txt.

[13] T"uys"uz and F. Alag"oz, - Satellite mobility pattern based handover management algorithm in LEO satellites,\| in Proc. IEEE ICC 2006, Istanbul,Turkey, June 2006.

[14] Ays, eg"ul T"uys"uz and Fatih Alag"oz, -Satellite Mobility Pattern Scheme for centrical and Seamless Handover Management in LEO Satellite Networksll, JOURNAL OF COMMUNICATIONS AND NETWORKS, VOL. 8, NO. 4, DECEMBER 2006

[15] M. Atiquzzaman, S. Fu, and W. Ivancic, -TraSH-SN: A transport layer seamless handoff scheme for space networks, in Proc. ESTC 2004, Palo Alto, CA, June 2004.

[16] X. Zhang, J. G. Castellanos, and A. T. Campbell, -Design and performance of mobile IP paging, $\| A C M$ Mobile Networks and Appl., vol. 7, no. 2, Mar.2002.

[17] A. G. Valko, —Cellular IP — a new approach to Internet host mobility, ACM Computer Commun. Rev., Jan. 1999

[18] Debabrata Sarddar, Arnab Raha, Tapas Jana, Shovan Maity, Utpal Biswas, and M.K. Naskar, -Reduction of Error in Handoff Initiation Time Calculation for NextGeneration Wireless Systems\| (IJCSE) International Journal on Computer Science and Engineering Vol. 02, No. 06, 2010, 2047-2052.

[19] H. N. Nguyen, S. Lepaja, J. Schuringa, and H. R. Van As, - Handover management in low earth orbit satellite IP networks,\| IEEE Global Telecommunications Conference, San Antonio, TX, USA, pp. 2730-2734, 25 29 November 2001

[20] E. Cayirci and I. F. Akyildiz, "User mobility pattern scheme for location update and paging in wireless systems," IEEE Trans. Mobile Computing, vol. 1, no. 3, pp. $236-247,2002$.

\section{AUTHORS PROFILE}

Soumya Das, son of Mr. Subrata Das and Mrs. Swapna Das, currently pursuing his B.Tech in Electronics \& Communication Engg. at Bengal Institute of Technology under West Bengal University of Technology. His research 
interest includes mobile communication \& satellite communication.

Debabrata Sarddar is currently pursuing his $\mathrm{PhD}$ at Jadavpur University. He completed his M.Tech in Computer Science \& Engineering from DAVV, Indore in 2006, and his B.Tech in Computer Science \& Engineering from Regional Engineering College, Durgapur in 2001. His research interest includes wireless and mobile communication

Dipsikha Ganguly, currently pursuing B.Tech in Electronics \& Communication engineering at B.P.Poddar Institute of Management \& Technology under West Bengal University of Technology. Her research interest includes wireless communication \& satellite communication.

Suman Kumar Sikdar completed his B.Tech in CSE from Murshidabad College of Engineering \& Technology and M.Tech in Computer Science \& Engineering from Jadavpur
University in 2011. His research interest includes wireless sensor networks and wireless communication systems.

Sougata Chakraborty is presently an employee of IBM (Kolkata). He has completed his B.Tech in IT from Murshidabad College of Engineering and Technology in 2008 and M.Tech in Computer Science \& Engineering from Jadavpur University in 2011. His research interest includes wireless sensor networks and wireless communication systems.

Kunal Hui is presently an employee of Cognizant (Kolkata). $\mathrm{He}$ has completed his B.Tech in Computer Science \& Engineering from Murshidabad College of Engineering and Technology (2004-2008) and M.Tech in Computer Science \& Engineering from Haldia Institute of Technology (20092011). His research interest includes wireless sensor networks and wireless communication systems 